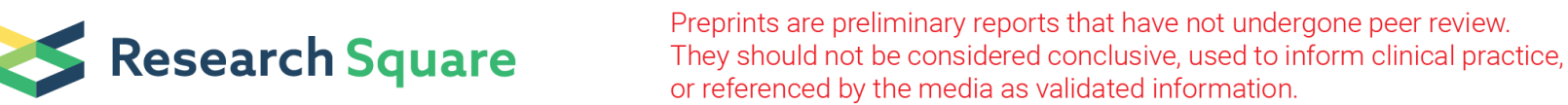

\section{Single-Use Digital Flexible Ureteroscopes as A Potential Choice for the Treatment of Lower Pole Renal Stones: A Multicenter Case-Control Study}

\section{Enguang Yang}

Institute of Urology, Lanzhou University Second Hospital

\section{Suoshi Jing}

Lanzhou University First Affiliated Hospital

\section{Yuanjie Niu}

Tianjin Medical University Second Hospital

Shiyong Qi

Tianjin Medical University Second Hospital

\section{Prabin Kumar Yadav}

Lanzhou University Second Hospital

\section{Li Yang}

Lanzhou University Second Hospital

Junsheng Bao

Lanzhou University Second Hospital

Junqiang Tian

Lanzhou University Second Hospital

Juan Wang

Lanzhou University Second Hospital

Ningchen Li

Peking University Shougang Hospital

\section{Tongwen Ou}

Xuanwu Hospital

\section{Zhiping Wang ( $\nabla$ wangzplzu@163.com )}

Institute of Urology, Lanzhou University Second Hospital

\section{Research article}

Keywords: renal calculi, lower pole, ureteroscopy, efficiency

Posted Date: November 6th, 2020

DOl: https://doi.org/10.21203/rs.3.rs-100944/v1 
License: (c) (i) This work is licensed under a Creative Commons Attribution 4.0 International License. Read Full License 


\section{Abstract}

Background: To compare the efficacy and safety of a single-use digital flexible ureteroscope (FURS) and a reusable FURS for the treatment of lower pole stones (LPS) smaller than $20 \mathrm{~mm}$.

Methods: A prospective case-control study was conducted using the clinical data from a multicenter, randomized, open-label clinical trial in four hospitals in China. A single-use digital FURS ZebraScope ${ }^{\mathrm{Tm}}$ was utilized in the trial group during surgery, with a reusable FURS URF-V used in the control group. The efficacy endpoints assessed were the 1-month postsurgical stone-free rate (SFR), operative time, length of postoperative hospital stay, and mean reduction in hemoglobin level. The safety outcomes assessed were the presence of adverse events (AEs), severe AEs (SAEs), and postoperative complications.

Results: In total, 49 patients with LPS underwent surgery using FURS. The demographic and preoperative parameters were comparable between the 2 groups. The 1-month SFR was $84.00 \%$ for the ZebraScope ${ }^{\mathrm{TM}}$ group and $58.33 \%$ for the URF-V group $(P<0.05)$. There was no difference between the two groups in the operative time $(P=0.665)$, the length of hospital stay $(P=0.308)$, the presence of postoperative complications $(P=0.307)$, the presence of AEs $(P=0.483)$ \and the presence of SAEs $(P=0.141)$.

Conclusions: This study demonstrates that single-use digital FURS is a safe and effective option and can offer higher SFR than the reusable FURS in the treatment of LPS smaller than $20 \mathrm{~mm}$. We recommend single-use digital FURS as an alternative to reusable FURS for the treatment of LPS.

Trial registration: The trial was registered in Chinese Clinical Trial Registry. The registration number: ChiCTR1900021615. Date of registration: 1/3/2019. This trial was registered retrospectively.

\section{Background}

Poor clearance of fragments and difficulty accessing lower pole calyces account for differences in treatment outcomes compared to stones in other locations in the kidney. It has been reported that the stone-free rate (SFR) after extracorporeal shock wave lithotripsy (SWL) for lower pole stones (LPS) is $25 \%$ $\sim 90 \%[1-3]$. The residual fragments of LPS still play a negative role in stone formation, which can become the core for further growth and formation of new stones[4].

The choice of treatment for patients with LPS remains controversial. SWL is a better choice for LPS less than $1 \mathrm{~cm}$ in diameter, while percutaneous nephrolithotomy (PCNL) is the first choice for LPS larger than $2 \mathrm{~cm}[5,6]$. For LPS $10 \sim 20 \mathrm{~mm}$ in size, Raman and Pearl recommended that PCNL is the best management, although ureteroscopy is an option in patients who are not considered candidates for PCNL[5]. Likewise, a survey of 205 urologists reported by Gerber revealed that $65 \%$ preferred SWL and $30 \%$ would advise PCNL[7].

Technical improvements, the introduction of a wide range of disposables, and improvements in digital imaging quality for flexible ureteroscope (FURS) have led to increased use and shorter operating times[4]. 
Grasso and Ficazzola[8] used FURS to treat LPS. The SFRs of LPS were $82 \%, 71 \%$ and $65 \%$ with stone sizes of less than $1 \mathrm{~cm}, 1 \sim 2 \mathrm{~cm}$ and greater than $2 \mathrm{~cm}$, respectively.

In recent years, single-use FURS was introduced and has been favored by urologists. Single-use FURS allows doctors to use laser lithotripsy with higher power and longer time. In addition, LPS is one of the high-risk factors for damage caused by the repeated use of FURS. Single-use FURS can reduce the psychological pressure on doctors caused by damage to medical equipment. Therefore, single-use FURS may have more advantages in the treatment of LPS. However, there are few clinical studies on whether single-use FURS has unique advantages over reusable FURS and can provide a higher SFR for LPS treatment. Therefore, we analyzed the data of LPS treatment using single-use FURS and evaluated the effectiveness and safety to provide the basis for clinical treatment of LPS.

\section{Methods}

\subsection{Data source and trial process}

A prospective case-control study was conducted using the clinical data from a multicenter, randomized, open-label, noninferiority clinical trial (registration number: ChiCTR1900021615) in four hospitals in China from September 2018 to February 2019. The clinical study was approved by the ethics committees of the Lanzhou University Second Hospital(2018-QX-029), the Second Hospital of Tianjin Medical University (2018K032), Peking University Shougang Hospital(IRB-2018-016-02), and Beijing Xuanwu Hospital(2018-007-1). Prior to the procedure, all patients signed an informed consent form and were enrolled in the study after a routine preoperative evaluation including urinalysis, urine culture, complete blood count, renal function, electrolyte determination, and nonenhanced computed tomography (CT). All procedures were performed by doctors experienced with the use of FURS. The patients were given general anesthesia and placed into the lithotomy position. A single-use digital FURS ZebraScope ${ }^{\text {TM }}$ was utilized in the trial group during surgery, with a reusable digital FURS (URF-V, Olympus, Tokyo, Japan) used in the control group. The structures and major parameters of the ZebraScope ${ }^{\mathrm{TM}}$ were introduced in our previous study[9].

\subsection{Inclusion and exclusion criteria}

The inclusion criteria were as follows:

(1) Maximum stone diameter of 6-20 mm confirmed by a CT scan.

(2) Stones located in the lower pole of the kidney.

(3) Adults aged 18 to 75 years.

The exclusion criteria were as follows:

(1) Coagulopathy or current anticoagulation therapy. 
(2) Anatomic abnormalities, such as horseshoe kidneys, calyceal diverticula, ureteral strictures, ureteropelvic junction obstructions, infundibular stenoses, and impassable urethral strictures.

(3) Comorbidities that interfere with participation in or completion of the study, for example, myocardial infarction, stroke, congestive heart failure, severe chronic respiratory lung disease, cancer, uncontrolled diabetes mellitus, pregnancy, mental illness, and severe systemic diseases or psychosis.

(4) Severe deformity of the hip joint that prevents meeting the requirement of the operation position.

(5) Patients who participated in other clinical trials within 3 months.

\subsection{Outcome assessment and statistical analysis}

The efficacy endpoints assessed were the 1-month postsurgical SFR, operative time, length of postoperative hospital stay and mean reduction in hemoglobin level. The safety outcomes assessed were the presence of adverse events (AEs), severe AEs (SAEs) and postoperative complications, including pain, fever, renal hematoma, hematuria, pelvicalyceal system injury, urinary sepsis, and steinstrasse formation. The operative time was defined as the time from ureteroscope insertion until the end of Foley catherization. "Stone-free" status was defined as no residual stone or stones $\leq 4 \mathrm{~mm}$ by plain abdominal radiograph of the kidneys, ureters, and bladder (KUB) and by CT scan at 1 month after stent removal. The radiologists who performed the CT scan and the KUB radiograph were totally blinded to the study objectives and protocols.

The data were analyzed by using Statistical Package for the Social Sciences, V. 22.0 (SPSS; SPSS Inc, Chicago, IL, USA). Continuous variables were compared by independent samples t-tests. Categorical variables were compared by the chi-square or Fisher's exact test, as appropriate. All analyses were planned as intention to treat.

\section{Results}

\subsection{Patients and stone characteristics}

Both groups were comparable. The patient and stone characteristics are shown in Table 1. The mean age of the patients in the trial group was 52.72 years, with a mean body mass index (BMI) of $25.32 \mathrm{~kg} / \mathrm{m}^{2}$. The mean age of the patients in the control group was 54.00 years, with a mean BMI of $26.47 \mathrm{~kg} / \mathrm{m}^{2}$. The mean stone sizes were 11.35 and $11.40 \mathrm{~mm}$ in the trial and control groups, respectively. The mean CT values were 927.56 and $967.53 \mathrm{Hu}$ in the trial and control groups, respectively. No significant differences were observed between the groups in terms of age, sex, stone size, CT value or procedural laterality. 
Table 1

Baseline demographics and clinical characteristics

\begin{tabular}{|c|c|c|c|}
\hline Characteristics & Trial group $(n=25)$ & Control group $(n=24)$ & P-value \\
\hline Age $(y)$, mean $\pm S D$ & $52.72 \pm 11.79$ & $54.00 \pm 12.69$ & 0.716 \\
\hline \multicolumn{4}{|l|}{ Sex, n (\%) } \\
\hline Male & $21(84 \%)$ & $19(79.17 \%)$ & 0.662 \\
\hline Female & $4(16 \%)$ & $5(28.83 \%)$ & \\
\hline $\mathrm{BMI}\left(\mathrm{kg} / \mathrm{m}^{2}\right)$, mean $\pm \mathrm{SD}$ & $25.32 \pm 3.92$ & $26.47 \pm 3.49$ & 0.282 \\
\hline $\mathrm{CT}$ value(Hu), mean $\pm \mathrm{SD}$ & $927.56 \pm 323.72$ & $967.53 \pm 279.35$ & 0.670 \\
\hline Stone size $(\mathrm{mm})$, mean \pm SD & $11.35 \pm 5.19$ & $11.40 \pm 4.53$ & 0.974 \\
\hline \multicolumn{4}{|l|}{ Procedural laterality, n (\%) } \\
\hline Left & $15(60.00 \%)$ & $15(62.5 \%)$ & 0.858 \\
\hline Right & $8(32.00 \%)$ & $6(25.00 \%)$ & 0.588 \\
\hline Bilateral & $2(8.00 \%)$ & $3(12.50 \%)$ & 0.603 \\
\hline
\end{tabular}

\subsection{Surgical outcomes}

The surgical outcomes are shown in Table 2. The 1-month SFR was $84.00 \%$ for the ZebraScope ${ }^{\text {TM }}$ group and $58.33 \%$ for the URF-V group ( $P \otimes 0.05)$. The mean operative times were 40.52 and $42.88 \mathrm{~min}$ in the trial and control groups, respectively $(P=0.665)$. The mean decreases in hemoglobin were 8.68 and $7.75 \mathrm{~g} / \mathrm{L}$ in the trial and control groups, respectively $(P=0.748)$. The mean lengths of hospital stay were 7.52 and 8.42 days in the trial and control groups $(P=0.308)$, respectively. The mean lengths of postoperative hospital stay were 1.76 and 2.08 days in the trial and control groups, respectively $(P=0.307)$. 
Table 2

Surgical outcomes comparison of the efficacy and safety between a single-use digital flexible ureteroscope (trial group) and a reusable digital ureteroscope (control group).

\begin{tabular}{|c|c|c|c|}
\hline Surgical outcomes & $\begin{array}{l}\text { Trial group }(n= \\
25)\end{array}$ & $\begin{array}{l}\text { Control group }(n= \\
24)\end{array}$ & $\begin{array}{l}\mathrm{P} \text { - } \\
\text { value }\end{array}$ \\
\hline Stone free rate, $\mathrm{n}(\%)$ & $21 / 25(84.00 \%)$ & $14 / 24(58.33 \%)$ & 0.047 \\
\hline Operative time (min), mean \pm SD & $40.52 \pm 17.63$ & $42.88 \pm 20.14$ & 0.665 \\
\hline $\begin{array}{l}\text { Mean drop in hemoglobin level(g/L), mean } \pm \\
\text { SD }\end{array}$ & $8.68 \pm 10.37$ & $7.75 \pm 9.72$ & 0.748 \\
\hline Hospital stay (d), mean \pm SD & $7.52 \pm 2.86$ & $8.42 \pm 3.23$ & 0.308 \\
\hline Postoperative hospital stays (d), mean \pm SD & $1.76 \pm 1.13$ & $2.08 \pm 1.06$ & 0.307 \\
\hline $\begin{array}{l}\text { Presence of postoperative complications, } n \\
\text { (\%) }\end{array}$ & $2(8 \%)$ & $0(0 \%)$ & 0.157 \\
\hline Adverse events, n (\%) & $8(32 \%)$ & $10(41.67 \%)$ & 0.483 \\
\hline Serious adverse events, $\mathrm{n}(\%)$ & $0(0 \%)$ & $2(8.33 \%)$ & 0.141 \\
\hline
\end{tabular}

Postoperative complications occurred in $2(8 \%)$ patients in the trial group and $0(0 \%)$ patients in the control group $(P=0.157)$. The AE rates were $32 \%$ and $41.67 \%$ in the trial and control groups, respectively $(P=0.483)$. The SAE rates were $0 \%$ and $8.33 \%$ in the trial and control groups, respectively $(P=0.141)$. AEs included hematuria, lower urinary tract symptoms (LUTS), fever, lung infection, and nausea. SAEs included ureteral stent migration and postrenal acute renal failure.

\section{Discussion}

Poor clearance of fragments and difficulty accessing lower pole calyces are challenges for the treatment of LPS. It has been reported that the SFR of LPS following ESWL ranges from $25-90 \%[1-3]$. The clearance of fragments of the LPS following ESWL is affected by three spatial anatomical factors, including the infundibulopelvic angle (IPA), infundibular width (IW), and infundibular length (IL)[10]. In addition, the measured value of the IPA correlated in a statistically significant manner with the overall success of flexible ureteroscopy for LPS[11]. Berkan et al.[12] evaluated 67 patients with LPS who received retrograde intrarenal surgery (RIRS), and the results showed that the lower pole anatomy, especially IPA, had a significant effect on the SFR of lower pole stones after RIRS. Studies conducted by Jessen et al.[13] showed that IPA and IL have adverse effects on SFR. Stephanie et al.[14] retrospectively reviewed 243 patients with LPS treated with FURS and analyzed the univariate and multivariate influencing factors of SFR. The results showed that the residual stone fragments were negatively related to the more acute IPA and the larger stone diameter.

Koo et al.[15] evaluated the clinical outcomes of reusable FURS compared with those of ESWL in 88 patients, and the results showed that the SFR of FURS was $59.4 \%$, which is similar to the present study. 
Bozzini et al.[16] prospectively evaluated the efficacy and safety of RIRS, ESWL and PCNL in the treatment of $1 \sim 2 \mathrm{~cm}$-sized LPS. The SFR was $82.1 \%$, and the complication rate was $14.5 \%$ after RIRS. Zhang et al.[17] showed that the SFR after FURS was $92 \%$, and the incidence of complications was $8.33 \%$ in the treatment of 1-2 cm LPS. Therefore, we found that the SFR after FURS treatment for LPS was similar to the above studies.

The 1-month SFR following a single-use ureteroscope was significantly higher than that after a reusable ureteroscope. The possible explanations are as follows. The single-use FURS used in the study can deflect $275^{\circ}$ in the upward and downward directions, and the deflection loss is small when the operating instruments are placed. To a certain extent, it can overcome the lithotripsy difficulty caused by acute IPA. Abdelsehid et al.[18] demonstrated that deflection was impaired when different instruments were placed in the working channels. With $200 \mu \mathrm{m}$ laser fiber or $365 \mu \mathrm{m}$ laser fiber, the FURS showed a decrease in deflection by $3.1 \% \sim 22.7 \%$ or $25.9 \% \sim 46.3 \%$. In the same case, single-use FURS outperformed fiber-optic FURS for all other settings in terms of deflection loss[19]. In addition, the outer diameter of the ZebraScope $^{\mathrm{TM}}$ is less than that of URF-V (8.7 Fr vs. 9.9 Fr). Higher irrigation flow and a clearer field of vision brought by a smaller outer diameter are favorable to the enhancement of operative efficiency and effect. Furthermore, the use of single-use products allowed doctors to boldly attempt procedures, without the concern of damage to reusable ureteroscopes with high purchase costs. LPS was one of the significant risk factors for FURS damage, which increased the psychological pressure on doctors.

Based on the present study, we propose the following suggestions for the treatment of LPS using FURS. First, if feasible, the LPS can be moved to the pelvis to lower the complexity barrier of lithotripsy. Second, it is necessary to evaluate the parameters of renal anatomy before surgery, including IPA, IW, and IL. For patients with LPS whose IPA of the involved kidney is acute, FURS with greater deflection should be selected. Third, the stones should be powdered as much as possible during surgery. For larger stone fragments, the nitinol basket should be used to extract or relocate from the lower calyx. In addition, combined with the physical stone removal method, the SFR after lithotripsy can be improved.

This study has several limitations. IPA, IL, and IW were not measured to assess the anatomical data of the target kidney before the operation and further follow-up was not conducted to evaluate the recurrence rates of LPS after FURS treatment. This result requires confirmation in an adequately powered prospective randomized controlled trial in patients with LPS.

\section{Conclusion}

Our study demonstrates that single-use digital FURS (ZebraScope ${ }^{\mathrm{TM}}$ ) is a safe and effective option and can offer higher SFR than the reusable digital FURS in the treatment of LPS smaller than $20 \mathrm{~mm}$. We recommend single-use digital FURS as an alternative to reusable digital FURS for the treatment of LPS.

\section{Abbreviations}


FURS: flexible ureteroscope

LPS: lower pole stones

SFR: stone-free rate

AEs: adverse events

SAEs: severe adverse events

SWL: extracorporeal shock wave lithotripsy

PCNL: percutaneous nephrolithotomy

CT: computed tomography

KUB: plain abdominal radiograph of the kidneys, ureters, and bladder

BMI: body mass index

LUTS: lower urinary tract symptoms

IPA: infundibulopelvic angle

IW: infundibular width

IL: infundibular length

RIRS: retrograde intrarenal surgery

\section{Declarations}

\section{Ethics approval and consent to participate}

The clinical study was approved by the ethics committees of the Lanzhou University Second Hospital(2018-QX-029), the Second Hospital of Tianjin Medical University (2018K032), Peking University Shougang Hospital(IRB-2018-016-02), and Beijing Xuanwu Hospital(2018-007-1). The study was conducted in accordance with the ethical standards of the Declaration of Helsinki. Written informed consent is required for study participation. All personal data are handled according to the federal law of data protection in China.

\section{Consent for publication}

Not applicable.

\section{Availability of data and materials}


The datasets used during the current study available from the corresponding author on reasonable request.

\section{Competing interests}

The authors declare that they have no competing interests.

\section{Funding}

This work is supported by the National Natural Science Foundation of China (NSFC: 81874088), the Fundamental Research Funds for the Central Universities (Izujbky-2018-kb14), Science and Technology Project of Tianjin (17ZXMFSY00060), the Education Commission Research Project of Tianjin (2017KJ208), and the Key Laboratory Fund Project of the Second Hospital of Tianjin Medical University (2017ZDSYS14). The funding body was not involved in study design, data extraction, analysis, and description, or manuscript writing.

\section{Authors' contributions}

WZP, NYJ, QSY participated in designing the protocol of the study. YEG, QSY, YL, BJS, TJQ, WJ, LNC, and OYW were responsible for data collection and management. YEG, JSS, and YPK were responsible for data analysis. YEG and YPK drafted the manuscript. JSS and WZP critically revised the manuscript. Each of the authors had carefully read and approved the final manuscript for publication.

\section{Acknowledgments}

None

\section{References}

1. Ozgor F, Sahan M, Yanaral F, Savun M, Sarilar O: Flexible ureterorenoscopy is associated with less stone recurrence rates over Shockwave lithotripsy in the management of 10-20 millimeter lower pole renal stone: medium follow-up results. Int Braz J Urol 2018, 44(2):314-322.

2. El-Nahas AR, Ibrahim HM, Youssef RF, Sheir KZ: Flexible ureterorenoscopy versus extracorporeal shock wave lithotripsy for treatment of lower pole stones of $10-20 \mathrm{~mm}$. BJU international 2012, 110(6):898-902.

3. Srivastava A, Zaman W, Singh V, Mandhani A, Kumar A, Singh U: Efficacy of extracorporeal shock wave lithotripsy for solitary lower calyceal stone: a statistical model. BJU international 2004, 93(3):364-368.

4. Turk C, Petrik A, Sarica K, Seitz C, Skolarikos A, Straub M, Knoll T: EAU Guidelines on Interventional Treatment for Urolithiasis. Eur Urol 2016, 69(3):475-482.

5. Raman JD, Pearle MS: Management options for lower pole renal calculi. Curr Opin Uro/ 2008, 18(2):214-219. 
6. Tiselius HG, Ackermann D, Alken P, Buck C, Conort P, Gallucci M: Guidelines on urolithiasis. Eur Urol 2001, 40(4):362-371.

7. Gerber GS: Management of lower-pole caliceal stones. J Endouro/ 2003, 17(7):501-503.

8. Grasso M, Ficazzola M: Retrograde ureteropyeloscopy for lower pole caliceal calculi. J Urol 1999, 162(6):1904-1908.

9. Qi S, Yang E, Bao J, Yang N, Guo H, Wang G, Li N, Cui X, Gao W, Ou T et al: Single-Use Versus Reusable Digital Flexible Ureteroscopes for the Treatment of Renal Calculi: A Prospective Multicenter Randomized Controlled Trial. J Endouro/ 2020, 34(1):18-24.

10. Ullah A, Zubair M, Khan N, Malik A: Frequency and Factors Effecting Non Clearance of Lower Pole Renal Stones. J Ayub Med Coll Abbottabad 2015, 27(2):384-387.

11. Geavlete P, Multescu R, Geavlete B: Influence of pyelocaliceal anatomy on the success of flexible ureteroscopic approach. J Endouro/ 2008, 22(10):2235-2239.

12. Resorlu B, Oguz U, Resorlu EB, Oztuna D, Unsal A: The impact of pelvicaliceal anatomy on the success of retrograde intrarenal surgery in patients with lower pole renal stones. Urology 2012, 79(1):61-66.

13. Jessen JP, Honeck P, Knoll T, Wendt-Nordahl G: Flexible ureterorenoscopy for lower pole stones: influence of the collecting system's anatomy. $J$ Endourol 2014, 28(2):146-151.

14. Dresner SL, Iremashvili V, Best SL, Hedican SP, Nakada SY: Influence of Lower Pole Infundibulopelvic Angle on Success of Retrograde Flexible Ureteroscopy and Laser Lithotripsy for the Treatment of Renal Stones. J Endourol 2020.

15. Koo V, Young M, Thompson T, Duggan B: Cost-effectiveness and efficiency of shockwave lithotripsy vs flexible ureteroscopic holmium:yttrium-aluminium-garnet laser lithotripsy in the treatment of lower pole renal calculi. BJU international 2011, 108(11):1913-1916.

16. Bozzini G, Verze P, Arcaniolo D, Dal Piaz O, Buffi NM, Guazzoni G, Provenzano M, Osmolorskij B, Sanguedolce $F$, Montanari $E$ et al: A prospective randomized comparison among SWL, PCNL and RIRS for lower calyceal stones less than $2 \mathrm{~cm}$ : a multicenter experience : A better understanding on the treatment options for lower pole stones. World journal of urology 2017, 35(12):1967-1975.

17. Zhang H, Hong TY, Li G, Jiang N, Hu C, Cui X, Chu C, Zhao JL: Comparison of the Efficacy of UltraMini PCNL, Flexible Ureteroscopy, and Shock Wave Lithotripsy on the Treatment of $1-2 \mathrm{~cm}$ Lower Pole Renal Calculi. Urol Int 2019, 102(2):153-159.

18. Abdelshehid C, Ahlering MT, Chou D, Park HK, Basillote J, Lee D, Kim I, Eichel L, Protsenko D, Wong B et al: Comparison of flexible ureteroscopes: deflection, irrigant flow and optical characteristics. $J$ Urol 2005, 173(6):2017-2021.

19. Marchini GS, Batagello CA, Monga M, Torricelli FCM, Vicentini FC, Danilovic A, Srougi M, Nahas WC, Mazzucchi E: In Vitro Evaluation of Single-Use Digital Flexible Ureteroscopes: A Practical Comparison for a Patient-Centered Approach. J Endourol 2018, 32(3):184-191.

\section{Supplementary Files}


This is a list of supplementary files associated with this preprint. Click to download.

- CONSORTChecklist.pdf 